\title{
Association of Knowledge and Attitude of Tuberculosis Among Patients in Ethiopia
}

\author{
Yenew Alemu Mihret \\ Department of Statistics, Injibara University, Injibara, Ethiopia \\ Email address: \\ yenewalemu@gmail.com
}

\section{To cite this article:}

Yenew Alemu Mihret. Association of Knowledge and Attitude of Tuberculosis Among Patients in Ethiopia. International Journal of Biomedical Engineering and Clinical Science. Vol. 5, No. 4, 2019, pp. 65-69. doi: 10.11648/j.ijbecs.20190504.12

Received: August 23, 2019; Accepted: November 5, 2019; Published: November 13, 2019

\begin{abstract}
Background: Tuberculosis is a disease of poverty, mainly affecting young adults in their most productive years. The aim of this study was to identify the association of knowledge and attitude of Tuberculosis in Ethiopia and to study the relationship between TB and independent variables. The source of data is obtained from 2011 EDHS. During the analysis stage, SPSS version 23, Stata version 14 and Microsoft-Excel are used as tools of analysis. Out of 14,104 households, only $4.2 \%$ households did not heard about TB. In general education level, current marital status, region and wealth index are significant factor to association of knowledge and attitude of tuberculosis among patients in Ethiopia.
\end{abstract}

Keywords: Knowledge, Attitude, Binary Logistic, Tuberculosis, Ethiopia

\section{Introduction}

Tuberculosis is a disease of poverty, mainly affecting young adults in their most productive years. For the first time in many years, recent World Health Organization data suggest that the absolute number of TB cases has been falling since 2006. Despite this achievement, TB continues to be one of the top causes of death among women ages 15-44, and in 2009 almost 10 million children were orphaned as a result of parental deaths caused by TB. The World Bank is committed to helping developing countries control and reduce the spread of TB.

Tuberculosis is a global public health priority, with 10.4 million people developing the disease in 2016 (10\% among HIV co-infected individuals), 1.67 million deaths and 490,000 multidrug-resistant plus an additional 110000 rifampicin-resistant cases [1]. Globally, in 2005 the annual incidence of TB expressed as the number of new TB cases was about 8.8 million people (7.4 million of these in Asia and sub-Saharan Africa), and 1.6 million deaths registered in annually [2].

Nationally, TB is the leading causes of morbidity and mortality. The incidence of TB of all forms and smearpositive TB is 341 and 152 per 100,000 populations, respectively. The prevalence and mortality of tuberculosis of all forms is estimated to be 546 and 73 per 100,000 populations, respectively [3].

Ethiopia ranks third in Africa and eighth among the 22 highest tuberculosis (TB) burdened countries in the world. The prevalence of all forms of TB is estimated at 261 per 100 000 population, leading to an annual mortality rate of 64 per 100000 population. The incidence rate of all forms of TB is estimated at 359 per 100000 population, while the incidence rate of smear-positive TB is 108 per 100000 population. The TB case detection rate, treatment success rate and TB cure rate are $74 \%, 82.5 \%$ and $67 \%$, respectively [4].

Tuberculosis (or TB) is an illness that is caused by a germ and mainly affects the lungs, but sometimes can affect other parts of the body. Sometimes you may not even feel sick or know you have TB disease. TB is usually spread from person-to-person by coughing. $\mathrm{TB}$ is curable and free treatment is available in Ethiopia [5].

As Ethiopia is one of the 22 Highest TB burden countries in the world, Federal Ministry of Health of Ethiopia is implementing TB Prevention and control program at all level of the health facility. Among both women and men who have heard about TB, the percentages who know that TB is spread through the air by coughing and that it can be cured increases steadily as levels of education and wealth increase. Rural women and men who have heard of TB are more likely than those in urban areas to want to keep secret a family member's TB infection. Women in SNNP and Gambela and men in 
SNNP are most likely to report that they would want to keep a family member's TB infection a secret. This proportion decreases with respondent's education and also tends to decrease with wealth, especially among men [6].

The objectives of this study is to identify the determinants of knowledge and attitude of Tuberculosis in Ethiopia and to study the relationship between TB and independent variables. Tuberculosis is silent dead disease in Ethiopia. Tuberculosis is showing a declining trend over the last decades. However, the rate is still very high and requires intervention to lower the death.

\section{Methods}

\subsection{Source of Data}

The source of data is obtained from 2011 EDHS. The 2011 Ethiopia Demographic and Health Survey is part of the worldwide MEASURE DHS project which is funded by the United States Agency for International Development. The survey was implemented by the Ethiopian Central Statistical Agency. This survey is the third in a series of similar surveys conducted at five-year intervals since 2000. During the analysis stage, SPSS version 23, Stata version 14 and Microsoft-Excel are used as tools of analysis. STATA was used in running the binary logistic regression and other analysis while Microsoft-Excel and SPSS were employed to generate numbers and sorting of data respectively.

\subsection{Variables in the STUDY}

Variables to be included in this study are selected from 2011 EDHS and conducted by the Central Statistics Agency of the Government of Ethiopia.

\subsubsection{Dependent Variable}

The variable whose value is influenced or to be predicted is called dependent (regressed or explained) variable. The dependent variable for this study is the prevalence (occurrence) of tuberculosis (no $=0$, yes $=1$ ).

\subsubsection{Independent Variables}

The variable which influences the values or is used for prediction, is called independent variable (regressor or explanatory). The variables that are considered in this research and expected to be the factors that influence the prevalence of TB in Ethiopia are types of place of residence (urban, rural), region (Tigray, Affar, Amhara, Oromiya, Somali, SNNP, Benshangul-gumuz, Gambela, Harari, Addis Ababa and Dire Dawa), wealth index (poorest, poorer, middle, richer and richest), education level (no education, primary, secondary and higher) and currently marital status (never in union, married, widowed, divorced and separated).

\subsection{Binary Logistic Regression}

There are different types of multivariate statistical techniques that can be used to predict a binary dependent variable from a set of independent variables. Among these, multiple linear regression analysis and discriminant analysis are two related techniques that quickly come to mind. However, these techniques bring difficulties when the dependent variable is dichotomous or categorical.

Even if linear discriminant analysis does allow direct prediction of group membership, assumption of multivariate normality of the independent variable as well as equal variance-covariance matrices in the two groups is required for the prediction rule to be optimal. Among these techniques the logistic regression model is used for the data analysis in this study.

For a binary response $Y_{j}$ and quantitative explanatory variable $X_{i j}, \mathrm{i}=1,2, \ldots, \mathrm{m}$ and $\mathrm{j}=1,2, \ldots, \mathrm{n}$.

Let $\pi_{j}=\mathrm{P}\left(X_{i j}\right)$ denote the "success probability" when $X_{i j}$ takes the values $x_{i j}$. The problem with linear model is that the probability model $\mathrm{E}(\mathrm{y})=\mathrm{X} \beta$ (where $\beta$ is the vector of parameters to be estimated) is used to approximate a probability value, $\pi_{j}=\mathrm{P}\left(Y_{j}=1\right)$ within the interval 0 and 1 , while $E\left(Y_{j}=1\right)$ is not so constrained. Therefore, we apply the logit transformation where the transformed quantity $\ln \left(\frac{\pi_{j}}{1-\pi_{j}}\right)$ lies in the interval $(-\infty, \infty)$ and it is modeled as

$$
\operatorname{Logit}\left(\pi_{j}\right)=\ln \left(\frac{\pi_{j}}{1-\pi_{j}}\right)==\beta_{0}+\beta_{1} X_{1 j}+\beta_{2} X_{2 j}+\ldots+\beta_{m} X_{m j}
$$

This implies

$$
\pi_{j}=\frac{\exp \left(\beta_{0}+\beta_{1} X_{1 j}+\beta_{2} X_{2 j}+\cdots+\beta_{m} X_{m j}\right)}{1+\exp \left(\beta_{0}+\beta_{1} X_{1 j}+\beta_{2} X_{2 j}+\cdots+\beta_{m} X_{m j}\right)}
$$

Where, the parameter $\beta_{i}$ determines the rate of increase or decrease of $X_{i j}$ on the $\log$ of odds that $\mathrm{Y}_{\mathrm{j}}=1$, controlling for other X's. Furthermore, $\exp \left(\beta_{i}\right)$ is the multiplicative effect on the odds of unit increase in $X_{i j}$, at fixed levels of the others X's.

\subsubsection{Methods of Parameter Estimation}

In logistic regression the parameters of the model are estimated using maximum-likelihood method. That is, the coefficients that make our observed results most "likely" are selected. Since the logistic regression model is non-linear, an iterative algorithm is necessary for parameter estimation.

The log likelihood $(\mathrm{L})$ is defined as

$$
\mathrm{L}(\beta)=\ln (\beta)=Y_{1} \ln \left(\pi_{1}\right)+\left(1-Y_{1}\right) \ln \left(1-\pi_{1}\right)+Y_{2} \ln \left(\pi_{2}\right)+\left(1-Y_{2}\right) \ln \left(1-\pi_{2}\right)+\ldots+Y_{n} \ln \left(\pi_{n}\right)+\left(1-Y_{n}\right) \ln \left(1-\pi_{n}\right)(3)
$$

The maximum likelihood estimates are the values of $\beta$ that maximize the above log-likelihood function. Through maximization of the log-likelihood function we can theoretically estimate the vector of parameter $\beta$. But the equation is nonlinear in $\beta$, and as a result the estimates do not have a closed form expression. Therefore, $\beta$ would be obtained by maximizing log-likelihood using iterative algorithm method [7].

\subsubsection{Model Adequacy Test}

The adequacy of fit or calibration of a model measures 
how well the model describes the response variable. Assessing goodness of fit involves investigating how the predicted values are closer to the observed values. The Hosmer-Lemeshow test is a statistical test for adequacy of fit for logistic regression models. The test is similar to a $x^{2}$ goodness of fit test and has the advantage of partitioning the observations into groups of approximately equal size, and therefore there are less likely to be groups with very low observed and expected frequencies.

A formula defining the calculation of $\mathrm{H}$ is as follows:

$$
\mathrm{H}=\sum_{i=1}^{g}\left(\frac{\left(O_{i}-E_{i}\right)^{2}}{n_{i} \widehat{p_{l}}\left(1-\widehat{p_{l}}\right)}\right)
$$

Where $\mathrm{O}_{\mathrm{i}}, \mathrm{E}_{\mathrm{i}}, \mathrm{n}_{\mathrm{i}}$, and $\widehat{p}_{\imath}$ denote the observed events, expected events, observations, and predicted risk for the $i^{\text {th }}$ risk deciles group. The test statistic asymptotically follows a

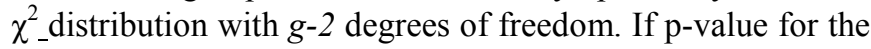
Hosmer-Lemeshow goodness-of-fit test is greater than 0.05 , we will not reject the null hypothesis that there is no difference between observed and model predicted values, implying that the model estimates are adequate to fit the data at an acceptable level or if the observed and expected numbers are sufficiently close, then we can assume that we have an adequate model.

\section{Results and Discussion}

\subsection{Results}

Descriptive statistics of all other variables are shown in table 1 . From the table 1 shows that 31 households from Tigray region don't have heard about tuberculosis (knowledge and attitude of tuberculosis) but 1353 households have heard about tuberculosis. In general, Out of 14,104 households, only $4.2 \%$ households did not heard TB.

The association between heard TB and other variable (chi square test) is shown in table 2.

From table 2 indicated there is association between the knowledge and attitude of tuberculosis and covariate variable. If the calculated p-value of the Hosmer-Lemeshow goodness-of-fit test statistic is greater than 0.05 , we do not reject the null hypothesis that there is no difference between observed and model-predicted values, implying that the model estimates are adequate to fit the data at an acceptable level (see table 3).

Based on table 4, Regarding the regional differences in $\mathrm{TB}$, the respondents from Affar, Oromiya, BenshangulGumuz, SNNP, harari, addis ababa and dire dawa and were $2.4,1.7,1.3,1.4,2.7,1.8$ and 3.1 times higher than knowledge and attitude of TB for households in Tigray, respectively, controlling for the other variables in the model. In the opposite, from Amhara, Somali and Gamebela are decresased by $5 \%, 30 \%$ and $15 \%$ as compared to Tigray region, respectively.

These findings show that respondents with higher, secondary and primary education were 5.4, 3.2 and 1.761 times more likely to have knowledge about TB than those with no formal education respectively. A similar trend was observed among those in wealth index. This results show that knowledge of TB for poorer, middle, richer and richest are $1.19,1.16,1.33$ and 1.65 times more likely to have knowledge of TB than those poorest households, respectively. When we look at the current marital status, the knowledge and attitude of TB for married, living with partner, windowed and divorced are increased by $68.5 \%$, $44.7 \%, 78.9 \%$ and $46.6 \%$ as compared to never in union households, respectively.

\subsection{Discussion}

In the present study, the risk factors for active TB amongst patients in Ethiopa were as follows: living in this region (Amhara, Tigray, Gambella, Addis ababa, Dire dawa and Harari, being unmarried and separated, being illiterate and being poorest households. The findings of the current study agreed with studies conducted in Affar, Oromiya, Somali, Benshangul-gumuz and S. N. N. P regional states of Ethiopia $[8,9]$.

We identified some factors that were associated with knowledge and attitude of TB. The level of education was one of the independent predictors for TB which is consistent with previous reports in Ethiopia [8, 10, 11], which concluded that high rates of illiteracy, low educational status were less knowledge and attitude towards TB. Similarly, in the present study, education level of the households were a significant risk factor for a TB, which is consistent with studies by [12-14] which is conducted in Nigeria.

\section{Conclusions}

The main objective of the study was to identify some of the factors that influence the knowledge and attitude of tuberculosis in Ethiopia. The study was based on secondary data obtained from the central statistical agency of Ethiopia. In general education level, current marital status, region and wealth index are significant factor to knowledge and attitude of tuberculosis in Ethiopia. Strategies to improve the knowledge and attitude about TB should focus on (Affar, Oromiya, Somali, Benshangul-Gumuz and SNNP) region, all education level, wealth index and those who are married, living with partner, windowed and divorced households in Ethiopia.

\section{Abbreviations}

TB: Tuberculosis

EDHS: Ethiopia Demographic and Health Survey

WHO: WHO World Health Organization

MTB: Mycobacterium tuberculosis

SNNP: Southern Nation Nationality of People

\section{Acknowledgements}

We would like to thank the staffs of Injibara University and Central statistical association especially my family for their invaluable support to enter into the prison system. 


\section{Appendix}

Table 1. Descriptive statistics.

\begin{tabular}{|c|c|c|c|}
\hline & & \multicolumn{2}{|c|}{ Heard of TB } \\
\hline & & No & Yes \\
\hline \multirow{11}{*}{ Region } & Tigray & 31 & 1353 \\
\hline & Affar & 27 & 973 \\
\hline & Amhara & 131 & 1834 \\
\hline & Oromiya & 108 & 1951 \\
\hline & Somali & 62 & 649 \\
\hline & Benishangul-Gumuz & 101 & 1038 \\
\hline & SNNP & 82 & 1617 \\
\hline & Gambela & 26 & 914 \\
\hline & Harari & 8 & 964 \\
\hline & Addis ababa & 5 & 1312 \\
\hline & Dire dawa & 5 & 913 \\
\hline \multirow{2}{*}{ Place of residence } & Urban & 33 & 4181 \\
\hline & rural & 553 & 9337 \\
\hline \multirow{4}{*}{ Education level } & No education & 314 & 4131 \\
\hline & Primary & 260 & 6409 \\
\hline & Secondary & 7 & 1619 \\
\hline & Higher & 5 & 1359 \\
\hline \multirow{5}{*}{ Wealth index } & poorest & 224 & 2621 \\
\hline & poorer & 122 & 1987 \\
\hline & middle & 115 & 2037 \\
\hline & richer & 88 & 2315 \\
\hline & richest & 37 & 4558 \\
\hline \multirow{6}{*}{ Currently marital status } & Never in union & 334 & 5316 \\
\hline & married & 229 & 7308 \\
\hline & Living with partner & 8 & 383 \\
\hline & windowed & 1 & 97 \\
\hline & divorced & 10 & 287 \\
\hline & separated & 4 & 127 \\
\hline
\end{tabular}

Table 2. The association between heard TB and other variable (chi square test).

\begin{tabular}{llll}
\hline & value & Degrees of freedom & Asymptotic significance(2-sided) \\
\hline Pearson chi-square (region vs TB) & 266.825 & 10 & 0.000 \\
TB vs place of residence & 171.563 & 1 & 0.000 \\
TB vs education level & 201.366 & 3 & 0.000 \\
TB vs wealth index & 251.431 & 4 & 0.000 \\
TV vs Current Marital status & 75.022 & 5 & 0.000 \\
\hline
\end{tabular}

Table 3. Hosmer and Lemeshow Test.

\begin{tabular}{llll}
\hline Step & Chi-square & df & Sig. \\
\hline 1 & 60.311 & 8 & 0.4087 \\
\hline
\end{tabular}

Table 4. Results of binary logistic regression analysis of households who have heard TB for selected covariates, EDHS 2011.

\begin{tabular}{lllllll}
\hline TB & Odds Ratio & Std. Err. & $\mathbf{z}$ & $\mathbf{P}>\mathbf{z}$ & [95\% C. I] & \\
\hline Region & & & & & & \\
Affar & 2.413404 & .2230519 & 9.53 & 0.000 & 2.01354 & 2.892677 \\
Amhara & .9549942 & .06975 & -0.63 & 0.528 & .8276209 & 1.101971 \\
Oromiya & 1.69991 & .1261119 & 7.15 & 0.000 & 1.469865 & 1.965958 \\
Somali & .7055351 & .0686471 & -3.58 & 0.000 & .5830402 & .8537657 \\
Benishan & 1.33778 & .1119018 & 3.48 & 0.001 & 1.135491 & 1.576106 \\
SNNP & 1.433483 & .1101313 & 4.69 & 0.000 & 1.233096 & 1.666436 \\
Gambela & .8581022 & .0770404 & -1.70 & 0.088 & .7196447 & 1.023199 \\
Harari & 2.650349 & .282118 & 9.16 & 0.000 & 2.151277 & 3.2652 \\
Addis Ab & 1.760052 & .1810813 & 5.49 & 0.000 & 1.438634 & 2.15328 \\
Dire Daw & 3.092926 & .3373807 & 10.35 & 0.000 & 2.497579 & 3.830185 \\
Place of residence & & & & & & \\
Rural & .9056217 & .0738767 & -1.22 & 0.224 & & \\
Education level & & & & & & \\
Primary & 1.757879 & .0802592 & 12.36 & 0.000 & 1.607407 & 1.062635 \\
Secondar & 3.201936 & .2599681 & 14.33 & 0.000 & 2.730882 & \\
Higher & 5.434922 & .5508318 & 16.70 & 0.000 & 4.455779 & 3.754244 \\
\hline
\end{tabular}




\begin{tabular}{|c|c|c|c|c|c|c|}
\hline TB & Odds Ratio & Std. Err. & $\mathbf{z}$ & $\mathbf{P}>\mathbf{Z}$ & {$[95 \%$ C. I $]$} & \\
\hline \multicolumn{7}{|c|}{ Wealth index } \\
\hline Poorer & 1.194019 & .0731683 & 2.89 & 0.004 & 1.058889 & 1.346394 \\
\hline Middle & 1.162724 & .0713374 & 2.46 & 0.014 & 1.030985 & 1.311297 \\
\hline Richer & 1.32806 & .0810395 & 4.65 & 0.000 & 1.178356 & 1.496783 \\
\hline Richest & 1.647444 & .1422466 & 5.78 & 0.000 & 1.39096 & 1.951221 \\
\hline \multicolumn{7}{|c|}{ Marital status } \\
\hline Married & 1.684982 & .0717087 & 12.26 & 0.000 & 1.550137 & 1.831556 \\
\hline Widowed & 1.788671 & .4159478 & 2.50 & 0.012 & 1.133933 & 2.821459 \\
\hline Divorced & 1.465728 & .1935536 & 2.90 & 0.004 & 1.131487 & 1.898703 \\
\hline separated & 1.280453 & .2696807 & 1.17 & 0.240 & .8474014 & 1.93481 \\
\hline cons & .4768491 & .0539244 & -6.55 & 0.000 & .3820524 & .5951672 \\
\hline
\end{tabular}

\section{References}

[1] World Health Organization. Global hepatitis report 2017. World Health Organization; 2017.

[2] Yadav SP, Mathur ML, Dixit AK. Knowledge and attitude towards tuberculosis among sandstone quarry workers in desert parts of Rajasthan. Indian Journal of Tuberculosis. 2006 Oct 1; 53 (4): 187.

[3] World Health Organization. The global MDR-TB \& XDR-TB response plan 2007-2008. Geneva: World Health Organization; 2007.

[4] World Health Organization. Global tuberculosis report 2013. World Health Organization; 2013.

[5] Alebachew Z, Kebede A, Tsegaye F. First Ethiopian National Population Based Tuberculosis Prevalence Survey

[6] Demographic E. Health Survey [EDHS] (2011). Central Statistical Agency. Addis Ababa, Ethiopia.

[7] Agresti, A. (2002). An Introduction to Categorical data Analysis. $2^{\text {nd }}$ Edition, John Wiley and Sons. Inc., New York.

[8] Bati J, Legesse M, Medhin G. Community's knowledge, attitudes and practices about tuberculosis in Itang special district, Gambella region, south western Ethiopia. BMC public health. 2013 Dec; 13 (1): 734.
[9] Tolossa D, Medhin G, Legesse M. Community knowledge, attitude, and practices towards tuberculosis in Shinile town, Somali regional state, eastern Ethiopia: a cross-sectional study. BMC public health. 2014 Dec; 14 (1): 804.

[10] Mesfin MM, Tasew TW, Tareke IG, Mulugeta GW, Richard MJ. Community knowledge, attitudes and practices on pulmonary tuberculosis and their choice of treatment supervisor in Tigray, northern Ethiopia. Ethiopian Journal of Health Development. 2005; 19 (I): 21.

[11] Kidane AH, Sifer D, Aklilu M, Pal M. Knowledge, attitude and practice towards human and bovine tuberculosis among high school students in Addis Ababa, Ethiopia. Int J Livest Res. 2015; 5: 1-1.

[12] Mushtaq MU, Majrooh MA, Ahmad W, Rizwan M, Luqman MQ, Aslam MJ, Siddiqui AM, Akram J, Shad MA. Knowledge, attitudes and practices regarding tuberculosis in two districts of Punjab, Pakistan. The international journal of tuberculosis and lung disease. 2010 Mar 1; 14 (3): 303.

[13] Mweemba P, Haruzivishe C, Siziya S, Chipimo P, Cristenson $\mathrm{K}$, Johansson E. Knowledge, attitudes and compliance with Tuberculosis treatment, Lusaka, Zambia. Medical Journal of Zambia. 2008; 35 (4).

[14] Okuonghae D, Omosigho S. Determinants of TB case detection in Nigeria: a survey. Global Journal of Health Science. 2010 Oct 1; 2 (2): 123. 